\title{
COMMENTARY
}

\section{Metformin overdose: time to move on}

\author{
Jean-Christophe Orban ${ }^{1,2}$, Eric Fontaine ${ }^{3}$ and Carole Ichai*1,2 \\ See related research by Protti et al., http://ccforum.com/content/16/5/R180
}

\begin{abstract}
Does metformin-associated lactic acidosis really exist? Despite an old controversy, there is no doubt about it. But do we understand what is going on? Laboratory findings raised several hypotheses explaining the pathophysiology of this disease. The main cause could be an inhibition of either gluconeogenesis or mitochondrial respiratory chain complex I. From bench to bedside, one hypothesis is now confirmed in humans. Metformin poisoning involves, at least partially, a mitochondrial dysfunction.
\end{abstract}

In this issue of Critical Care, Protti and colleagues [1] report the effects of metformin on human platelets both in vitro and ex vivo. In vitro experiments were performed on healthy platelets incubated with increasing doses of metformin, whereas ex vivo experiments were done on platelets from patients presenting accidental metformininduced lactic acidosis. In both situations, platelets' lactate production and mitochondrial functions were measured. In vitro, a dose-dependent relationship between metformin concentration and lactate production was found. In both conditions, high levels of metformin decreased mitochondrial respiratory chain complex I activity, mitochondria polarization, and oxygen consumption. Ex vivo only, mitochondria respiratory chain complex IV activity declined.

Metformin is a biguanide that has been used as a firstline drug for type 2 diabetes treatment since 1957 in Europe and 1995 in the US [2]. Metformin was reputed to induce lactic acidosis, partly because phenformin, another biguanide, was withdrawn from the market because of an unacceptable rate of this complication [3]. However, numerous clinical studies reported a similar incidence of lactic acidosis in diabetic patients with or without

\footnotetext{
*Correspondence: ichai@unice.fr

'Service de Réanimation Médico-chirurgicale, Hôpital Saint-Roch, Centre Hospitalier Universitaire de Nice, 5 rue Pierre Dévoluy, 06006 Nice, France Full list of author information is available at the end of the article
}

metformin, leading some authors to deny the existence of metformin-associated lactic acidosis [4]. However, in usual clinical practice, metformin contraindications are not often respected [5]. Moreover, physicians do not really monitor adequately their prescription. As a result, numerous publications reported the association between metformin and lactic acidosis [6,7]. When a cause of lactic acidosis such as shock state or acute renal failure is present, the responsibility of metformin could be questioned. But when healthy patients without risk factors develop metformin poisoning leading to lactic acidosis, there is no doubt about this link. However, metformin inhibits hepatic gluconeogenesis in different animal species and decreases mitochondrial respiratory chain complex I activity in different organs [8,9]. Both conditions can lead to lactate accumulation. Until recently, the clinical research on metformin-associated lactic acidosis was limited to retrospective studies describing incidence, risk factors, and supportive treatments. A big step forward was made when the Gattinoni group [10] reported a decrease in oxygen consumption after metformin poisoning in humans, strongly suggesting that metformin was able to induce mitochondrial dysfunction in humans. The study by Protti and colleagues elegantly confirms the implication of mitochondria in the pathophysiology of this disease. But it does not rule out the effects of metformin on gluconeogenesis. Further research is needed to assess the respective parts of these mechanisms.

Of course, the importance of platelet mitochondrial dysfunction per se has to be put in perspective. Platelets are probably not involved in lactic acidosis build-up during metformin overdose. However, as demonstrated previously in the pig, platelet mitochondrial dysfunction mirrors the mitochondrial dysfunction in other vital organs [11]. Platelets are more easily accessible than vital organs like the liver or kidney. For research purposes in humans, this approach seems to be promising to evaluate the effects of potential therapies. However, a possible limit to their findings lies in their model. It is not clear whether this model represents acute or chronic overdose. This question is important as they are considered different conditions with different prognoses [12]. Acute intentional poisoning clearly has a better outcome than accidental accumulation. 
Now that serious research on this rare disease has started, we can also imagine improving its care. Currently, the treatment is only supportive: increasing blood pressure with fluid infusion and catecholamines and promoting metformin elimination by renal replacement therapy. Restoring ATP production during energy failure due to mitochondria dysfunction is still challenging. New ideas could come from metabolic manipulations. In severe sepsis, another condition associated with mitochondrial dysfunction, succinate can bypass respiratory chain complex I inhibition and restore oxygen consumption [13]. In isolated cells, succinate is reputed not to cross the plasma membrane, but methyl succinate (a cellpermeant succinate) has been used to bypass metformin blockade of respiratory chain complex I [14]. This intervention led to a reduction of metformin toxicity. This strategy might be a therapeutic modality for metformin overdose in the future.

\section{Competing interests}

The authors declare that they have no competing interests.

\section{Author details}

'Service de Réanimation Médico-chirurgicale, Hôpital Saint-Roch, Centre Hospitalier Universitaire de Nice, 5 rue Pierre Dévoluy, 06006 Nice, France. ${ }^{2}$ IRCAN, Faculté de Médecine, Université de Nice, Avenue de Valombrose, 06107 Nice, France. ${ }^{3}$ INSERM, U1055, 2280 rue de la piscine, 38041 Grenoble, France.

Published: 25 October 2012

\section{References}

1. Protti A, Lecchi A, Fortunato F, Artoni A, Greppi N, Vecchio S, Fagiolari G, Moggio M, Comi GP, Mistraletti G, Lanticina B, Faraldi L, Gattinoni L: Metformin overdose causes platelet mitochondrial dysfunction in humans. Crit Care 2012, 16:R180.

2. UK Prospective Diabetes Study (UKPDS) Group: Effect of intensive blood-glucose control with metformin on complications in overweight patients with type 2 diabetes (UKPDS 34). Lancet 1998, 352:854-865.

3. Kwong SC, Brubacher J: Phenformin and lactic acidosis: a case report and review. J Emerg Med 1998, 16:881-886.

4. Salpeter SR, Greyber E, Pasternak GA, Salpeter EE: Risk of fatal and nonfatal lactic acidosis with metformin use in type 2 diabetes mellitus. Cochrane Database Syst Rev 2010, (4):CD002967.

5. Holstein A, Nahrwold D, Hinze S, Egberts EH: Contra-indications to metformin therapy are largely disregarded. Diabet Med 1999, 16:692-696.

6. Orban JC, Giunti C, Levraut J, Grimaud D, Ichai C: Metformin-associated lactic acidosis remains a serious complication of metformin therapy. Ann Fr Anesth Reanim 2003, 22:461-465.

7. Wills BK, Bryant SM, Buckley P, Seo B: Can acute overdose of metformin lead to lactic acidosis? Am J Emerg Med 2010, 28:857-861

8. Foretz M, Hébrard S, Leclerc J, Zarrinpashneh E, Soty M, Mithieux G, Sakamoto K, Andreelli F, Viollet B: Metformin inhibits hepatic gluconeogenesis in mice independently of the LKB1/AMPK pathway via a decrease in hepatic energy state. J Clin Invest 2010, 120:2355-2369.

9. El-Mir MY, Nogueira V, Fontaine E, Avéret N, Rigoulet M, Leverve X: Dimethylbiguanide inhibits cell respiration via an indirect effect targeted on the respiratory chain complex I. J Biol Chem 2000, 275:223-228.

10. Protti A, Russo R, Tagliabue P, Vecchio S, Singer M, Rudiger A, Foti G, Rossi A, Mistraletti G, Gattinoni L: Oxygen consumption is depressed in patients with lactic acidosis due to biguanide intoxication. Crit Care 2010, 14:R22.

11. Protti A, Fortunato F, Monti M, Vecchio S, Gatti S, Comi GP, Giuseppe RD, Gattinoni L: Metformin overdose, but not lactic acidosis per se, inhibits oxygen consumption in pigs. Crit Care 2012, 16:R75.

12. Seidowsky A, Nseir S, Houdret N, Fourrier F: Metformin-associated lactic acidosis: a prognostic and therapeutic study. Crit Care Med 2009, 37:2191-2196.

13. Protti A, Carré J, Frost MT, Taylor V, Stidwill R, Rudiger A, Singer M: Succinate recovers mitochondrial oxygen consumption in septic rat skeletal muscle. Crit Care Med 2007, 35:2150-2155.

14. Hinke SA, Martens GA, Cai Y, Finsi J, Heimberg H, Pipeleers D, Van de Casteele M: Methyl succinate antagonises biguanide-induced AMPK-activation and death of pancreatic beta-cells through restoration of mitochondrial electron transfer. Br J Pharmaco/ 2007, 150:1031-1043.

doi:10.1186/cc11664

Cite this article as: Orban J-C, et al.: Metformin overdose: time to move on. Critical Care 2012, 16:164 